\title{
GENIUS LOCI: BILD - STIMMUNG - ERINNERUNG. DIE KLAVIERKOMPOSITIONEN LEOŠ JANÁČEKS IM EUROPÄISCHEN KONTEXT
}

I.

In einem Brief vom 18. November 1906 beklagte sich Leoš Janáček bei Jan Bramberger, dem Redakteur der Zeitschrift Smetana, über eine Prager Aufführungskritik seiner Komposition Vater unser und stellte abschließend fest: „Verzeihen Sie, daß ich mich beschwere, denn ich weiß, Sie werden zustimmen, dass jedes Werk so aufgeführt werden soll, wie es gemeint war-aber daß es auch nach seiner Wahrheit beurteilt werde. ${ }^{\text {"1 }}$ Dieser Satz hat zu mancherlei Interpretationen und Spekulationen in einem Lebensabschnitt des Komponisten geführt, in der er in einer Krise, in Phasen des Suchens, des Umdenkens und einer persönlichen wie künstlerischen Neuorientierung befangen war. Was bedeutet für Janáček „Wahrheit“ in der Musik? Ist es für ihn ein unverwechselbarer Personalstil, neue Impulse, auf einem historischen Fundament gegründet, das Nach-außen-kehren individueller Befindlichkeit und Empfindungen als künstlerischer Ausdruck, die Einbindung nationaler Identität, der Lebensstationen, der selbst erlebten politischen Ereignisse als Genius loci für sein Denken und Schaffen?

Dass diese möglichen Antworten gerade im Zusammenhang und bei der Beschäftigung mit seinem Klavier-CEuvre auftauchen, kommt nicht von ungefähr. Denn das erste Jahrzehnt des 20. Jahrhunderts, in dem die drei Solowerke Po zarostlém chodnícku (Auf verwachsenem Pfade), zwei Sätze der Sonáta 1. X. 1905 und $V$ mlhách (Im Nebel) entstanden sind, wird für Janáček zu den entscheidenden Jahren seines Lebens. Im Folgenden sollen diese drei Zyklen aus unterschiedlichen Perspektiven erläutert und gedeutet werden: Der biografische Ansatz zeigt Einflüsse eines Genius loci seiner mährischen Heimat, der Stadt Brno wie der politischen Ereignisse. Der musikhistorische Hintergrund wird von der Entwicklung von Kompositionsstrukturen und der Klaviermusik im Verlauf

1 Leoš Janáček in Briefen und Erinnerungen. Ausgewählt, mit Beiträgen und Anmerkungen versehen von Bohumír Štědroň. Prague, 1955, S. 80. 
des 19. Jahrhunderts bestimmt. Die Analyse des 1912 veröffentlichten Zyklus Im Nebel wird schließlich die Ergebnisse zusammenführen und bewerten.

II.

Die Janáček-Forschung ist sich darüber einig, dass die Zeit zwischen dem 50. und dem beginnenden 60. Lebensjahr von persönlichen Krisen und schöpferischen Umbrüchen gekennzeichnet war. Opernprojekte fanden in Prag und Brünn nicht die Resonanz und Anerkennung, die sich der Komponist erwartet hatte. Das Werk Osud (Schicksal) scheiterte an einem unzulänglichen Libretto, an Zwistigkeiten für eine mögliche Aufführung in Brünn, verschwand zu Beginn des I. Weltkrieges in der Schublade und kam erst 1958 an die Öffentlichkeit. Jenůfa wurde zwar im Januar 1904 in Brünn uraufgeführt, fand aber wenig Zustimmung. Erst nach mehreren Umarbeitungen führte eine Aufführung 1916 in Prag zum Durchbruch der Oper und machte Janáček als Komponisten bekannt. Sein Wirken in der mährischen Hauptstadt als Chordirigent, Pädagoge, als Redakteur sowie als Sammler und Arrangeur von Volksliedern seiner Heimat schien ihn kaum zu befriedigen. „Ich bin hier in Brünn ein armer Kerl - wie in der Wüste - wo anständige Musik nicht zu hören ist", schrieb Janáček in einem Brief vom 9. Oktober 1903. ${ }^{2} 1904$ bewarb er sich dann vergeblich um den Direktorposten des Konservatoriums in Warschau.

Es ist bezeichnend, dass sich der Komponist gerade jetzt einer anderen $\mathrm{Mu}-$ sikgattung zuwendet, die ihm - so scheint es - mehr Befriedigung bei seinen künstlerischen Ausdrucksmöglichkeiten verschaffen und ihn aus Resignation und Frustration herausholen könnte. Zwischen 1901 und 1908 entstanden zehn Klavierstücke, ursprünglich teilweise für Harmonium, das obligate Instrument jedes Dorfschullehrers, konzipiert. Die einzelnen Stücke waren zunächst nicht mit einer Überschrift versehen. 1911 erschien der vollständige Zyklus im Druck mit dem programmatischen Titel Po zarostlém chodničku (Auf verwachsenem Pfade). Der Titel weist wie bei den beiden weiteren Werken für Klavier solo auf Intentionen hin, die durch die einzelnen Überschriften der Stücke noch konkreter in Erscheinung treten. Der Komponist begibt sich auf einen ihm vertrauten Pfad, der im Laufe der Jahre zugewachsen ist, auf einen Weg zurück in die eigene Vergangenheit. Es ist die Zeit seiner Jugend in Hukvaldy und der späteren alljährlichen Sommerfrische im heimatlichen mährischen Dorf. Ereignisse, Stimmungen, Erinnerungen tauchen auf, freudvoll glückliche, aber auch bedrückend schmerzhafte, die sich zu klingenden Bildern verdichten: Tages- und Jahreszeiten, schwatzende Frauen am Dorfbrunnen, eine Wallfahrt zur Muttergottes von Frydek und insbesondere der allmählich zur Gewissheit werdende Tod seiner

2 Brief an Kamilla Urválek vom 9. Oktober 1903, in Leoš Janáček in Briefen und Erinnerungen, op. cit., S. 95. 
Tochter Olga. Es sind schlichte dreiteilige Miniaturen ohne pianistische Ambitionen, von melancholischer Lyrik bestimmte persönliche Bekenntnisse, die zuweilen wie eine geschlossene dramatische Szene auf den Hörer wirken. Was nach außen wie eine aus einem momentanen Einfall geborene Improvisation erscheint, erweist sich seiner inneren Struktur nach als bewusst gestaltetes artifizielles Spiel mit Motiven, deren Varianten und thematischen Bezügen. Der Prager Musikologe Jaroslav Jiránek hat sie in einer beeindruckenden Analyse als semantisches Ineinanderwirken von Gegenwart und Vergangenheit gedeutet, als „,innere Antithesen“ von „Musikbildern“ und zugleich als „Kunst der Dethematisierung und Thematisierung der instrumentalen Figuration". ${ }^{3}$ Hier bricht sich ein neuartiger Klavierstil Bahn, der sich auch in weiteren Werken dieses Genre wiederfindet, generell Janáčeks spätere Musiksprachlichkeit bestimmen wird.

Die zwei Sätze mit dem späteren Zusatz Sonate und der Bezeichnung Von der Straße - 1. Oktober 1905 - dokumentieren den kompositorischen Reflex auf ein politisches Ereignis in Brünn, das den Patrioten Janáček stark erschüttert hatte. Im Zusammenhang mit Auseinandersetzungen um kulturelle Belange zwischen tschechischen und deutschen Volksgruppen gab es an diesem 1. Oktober einen Protestmarsch mit Demonstrationen und Gegendemonstrationen, die ihren traurigen, gewalttätigen Höhepunkt vor dem Vereinshaus Besední dům fanden: Ein junger Mann kam dabei ums Leben. Auf dem Titelblatt der Komposition, die Ende Januar 1906 im Besedni dům erstmals aufgeführt wurde, stand zu lesen:

Die weißen Marmorstufen der Beseda in Brünn -

Von Blut überströmt sinkt nieder der einfache Arbeiter Frant.[išek] Pavlik Er kam nur, entflammt für die Hochschule, und wurde von rohen Mördern erschlagen.

Leoš Janáček

Dem Andenken des bei den Manifestationen

für die Universität in Brünn

ermordeten Arbeiters. ${ }^{4}$

Den dritten Satz des Werkes, vermutlich ein ausgedehnter Trauermarsch, vernichtete der Komponist noch vor der öffentlichen Aufführung. Die beiden anderen - Vorahnung (Con moto) und Tod (Adagio) - erklangen ein weiteres Mal im privaten Kreis in Prag. Auch diese Sätze vernichtete Janáček danach, sie sind nur durch eine Abschrift der Brünner Pianistin Ludmilla Tučková erhalten geblieben.

JIRÁNEK, Jaroslav. Janáčeks Klavierkompositionen vom Standpunkt ihres dramatischen Charakters. Versuch einer semantischen Analyse. Archiv für Musikwissenschaft XXXIX, 1982, S. 179-197; zur Begrifflichkeit S. 187.

4 SAREMBA, Meinhard. Leoš Janáček. Zeit - Leben - Werk - Wirkung. Kassel / Basel etc., 2001, S. 207. 
Auch in diesem ,Restwerk' dominiert zusammen mit einer latent vorhandenen Sonatenform ebenfalls die kompositorische Arbeit mit knappen prägnanten Motiven und ihren variativen Ableitungen, strukturell und semantisch verknüpft mit Vorahnung und Tod des jungen Mannes. Dazu kommt eine emotional berührende Zerrissenheit der Stücke, die die seelische Erschütterung des Komponisten zu spiegeln scheint.

III.

Bevor auf den letzten Zyklus $V$ mlhách (Im Nebel) genauer eingegangen wird, soll gleichsam als Rückblende auf Janáčeks mögliche Anregungen und Vorbilder seines motivorientierten Klavierstils eingegangen werden. Chopin und vor allem Dvořák scheint er verehrt zu haben, von direkten Beeinflussungen durch bestimmte Komponisten will er jedoch nichts wissen: „Haben wir bei uns noch nicht genug solcher Mahler, Strauss, Schönberg und Debussy? Läuft ihnen doch alles nach!“,, 5 erfahren wir, und: „,Vorwärts' ist bei mir nicht erlernt, sondern

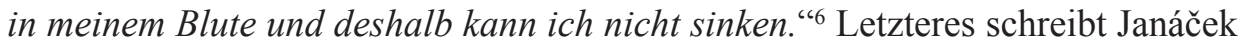
in einem Brief vom 4. Februar 1880 zu einem Zeitpunkt, als der Fünfundzwanzigjährige gerade seine Studienzeit am Konservatorium in Leipzig beendete und gleich darauf in Wien fortsetzte. Das „Vorwärts“ eines in Brünn bereits bekannten praktischen Musikers bezog sich demnach auf eine ihm wichtige Erweiterung seiner pianistischen wie auf die Intensivierung kompositorischen Fertigkeiten. Janáček nimmt bei Leo Grill in Leipzig zusätzliche, kostspielige Privatstunden, weil dieser , die Vergrößerungen und Erweiterungen der musikalischen Formen sonst nicht in der Schule vorträgt, [...] und doch ist gerade dieses sehr nötig für einen jeden, der komponieren will, da dadurch dem Geist ein Weg gezeigt wird, wo er wirklich und sicher gutes und doch neues schaffen kann, denn sonst ist

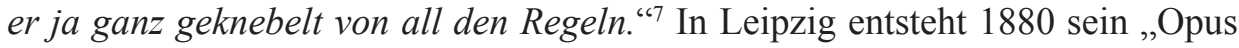
1“, Thema con variazioni für Klavier, die er seiner Verlobten und späteren Frau Zdenka Schulz zueignet. In Wien widmet er sich noch einmal dem Klavier- und Kompositionsstudium, von seinem dortigen Kompositionslehrer Franz Krenn zeigt er sich jedoch enttäuscht. Er sei „ein Mensch mit guten Ansichten, aber zu wenig gründlicher Kenntnis. Ich will auch nur weiter auf Grundlage Grills [in Leipzig] arbeiten und mich nicht durch die Wagner 'schen Schwülste beeinflussen lassen." ${ }^{\circ 8}$ Krenn sei „alt", er blicke „spottend [...] auf alle Regeln des strengen Stils [herab], die doch immer und immer die Grundlage einer soliden Arbeit bil-

5 TYRRELL, John (Hrsg.). My Life with Janáček - The Memoirs of Zdenka Janáčková. London, 1998, S. 128. Zitiert nach Saremba, Leoš Janáček, S. 219.

6 Ibid., S. 156 (S. 219).

7 Brief vom 1. Februar 1880, in KNAUS, Jakob. Intime Briefe 1879/80 aus Leipzig und Wien. Zürich, 1985, S. 160. Zitiert nach Saremba, Leoš Janáček, S. 73.

Brief vom 3. April 1880, ibid. (Anm. 7), S. 199 (S. 74). 
den müssen." Janáček bricht nach wenigen Monaten sein Wiener Studium ab; dennoch stellt ihm sein Lehrer ein ermutigendes Zeugnis aus: „Mit Vergnügen erkläre ich, dass ich Herrn Leoš Janáček beim Unterrichten im Konservatorium als einen sehr gebildeten Musiker kennen gelernt habe, welcher nicht nur das Gebiet der Harmonielehre, sondern auch jenes der kontrapunktischen und freien Formen mit vollkommener Sicherheit beherrscht, und daher auf Grundlage dessen zu schönsten Hoffnungen berechtigt. “"10

Die wenigen Briefzitate und Quellen lassen den Schluss zu, dass es dem jungen Komponisten vorrangig um drei Dinge ging: um die Vervollkommnung der erlernten kompositorischen Formen, um ein Vorwärtsblicken auf der Suche nach neuen Wegen und - daraus resultierend - um eine umfassende musikalische Bildung. Was sein Klavierschaffen betrifft, das 1880 mit schulmäßigen Variationen eines Themas begonnen hatte und 1912 mit den vier Stücken Im Nebel abgeschlossen sein wird, so kann ihm ein musikhistorische Wandel der Gattung Klaviermusik seiner nationalen wie europäischen Zeitgenossen ebenso wenig entgangen sein. Komponisten der Romantik wie Schumann und Chopin wenden sich von der Sonate als Form und Gattung ab und bevorzugen in zunehmendem Maße kleinformatige einsätzige Kompositionen, auch als „Charakterstücke“ oder „lyrische Klavierstücke" bezeichnet und in Zyklen zusammengefasst als Moments musicaux und Impromptus bei Schubert bis zu Nocturnes und Nachtstücken bei Chopin oder Schumann. ${ }^{11}$ Programmatische Überschriften und Titel lassen kaum auf bestimmte Formmuster oder -prinzipien schließen, sondern gelten als musikalische Miniaturen ohne Textbezug mit frei zu assoziierenden geistvollen „Inhalten“, ähnlich einer Phantasie. Sie ,fungierten als eine Art musikalischen Feuilletons, als kompositorische Diskussionsbeiträge, zu denen die Leser bzw. Spieler Stellung beziehen und an denen sie ihre eigene Urteilskraft erproben und schulen sollten." ${ }^{212}$ Schumann assoziiert bei einem Impromptu Schuberts ein „Nachdenken an Vergangenes“, daraus will Arnfried Edler „,den Rückblick auf die entschwindende Gattung Sonate überhaupt ${ }^{* 13}$ ableiten, eine Gattung, die in der Tat gegenüber den Charakterstücken des Komponisten in eine Krise geraten ist. Dabei spielen auch die gesellschaftlichen Schichten des Adels und des gehobenen Bürgertums eine wesentliche Rolle und - damit verbunden - ein Rückzug dieser Art von Musik aus den öffentlichen Konzertveranstaltungen in die mehr private Sphäre eines Hauses oder Salons. Charakterstücke verbinden bildhafte, auch poetische Überschriften oder Sujetbezeichnungen zumindest assoziativ für

9 Brief vom 6. April 1880, ibid., S. 203 (S. 75).

Ibid., S. 239 (S. 76/77).

11 Vgl. JUNG, Hermann. Musik des ,traurigen Lächelns' - ein Virtuose wird zum Poeten. Zu den Nocturnes von Frédéric Chopin. In JUNG-KAISER, Ute - KRUSE, Matthias (Hrsg.). Chopin, der Antistar. (= Wegzeichen Musik 5). Hildesheim / Zürich etc., 2010, S. 35-65, bes. S. $42-44$.

12 EDLER, Arnfried. Gattungen der Musik für Tasteninstrumente. (= Handbuch der musikalischen Gattungen 7,3). Laaber, 2003, S. 240. 
Spieler und Hörer mit seelischen Befindlichkeiten des Menschen. Solche musikalischen Miniaturen mit semantischen Anteilen finden sich auch bei Janáčeks nationalen Zeitgenossen, so bei Smetana in einigen Charakterstücken, bei Dvořák in den Humoresken und den Poetischen Stimmungsbildern oder bei Fibich, der ebenfalls in Leipzig studierte, in der umfangreichen Sammlung Stimmungen, Eindrücke, Erinnerungen.

Auch die inneren kompositorischen Strukturen wandeln sich. Bereits bei den Klaviersonaten von Schubert lässt sich ein Abrücken von der thematischen Arbeit hin zu motivischer Variantentechnik erkennen. ${ }^{14}$ Im symphonischen Bereich arbeitet Franz Liszt in seinen Symphonischen Dichtungen wie auch in der Klaviersonate mit Motivtransformationen, die von einem motivischen Kern ausgehen und sich über die gesamte Komposition ausbreiten. Für Wagner scheinen solche Motivtransformationen Impulsgeber für seine sogenannte Leitmotivtechnik gewesen zu sein als Verbindung von musikalisch strukturellen Elementen und wortsprachlicher Semantik. Nicht zuletzt hat Schönberg in seiner freitonalen Phase mit den Sechs kleinen Klavierstücken op. 9, niedergeschrieben 1911 und 1912 uraufgeführt, versucht, den durch den Wegfall von Tonalität und Harmonik fehlenden formalen Zusammenhalt mit Motiv- und Figurationstechnik auszugleichen, um danach zur Dodekaphonie weiterzuschreiten. „Minimalismen“ in mitunter extremer Ausprägung finden sich auch bei seinem Schüler Anton Webern.

\section{IV.}

Eben in diesem Jahr 1912 beendete Janáček in Brünn seinen Zyklus Im Nebel. Der Druck erfolgte im darauf folgenden Jahr, Marie Dvořáková spielte die Uraufführung im Januar 1914 in der Brünner Orgelschule. Es kann bei den musikhistorisch wie musiktheoretisch hochqualifizierten Bildung Janáčeks, seinen Kenntnissen und Erfahrungen aus der Praxis kaum möglich sein, dass der Komponist durch die angesprochenen Wandlungen innerhalb des Komponierens nicht beeinflusst war, sie zumindest unbewusst in sein Klavierwerk hat einfließen lassen. Außer der Zyklus-Bezeichnung wird in diesem Werk auf erläuternd programmatische Überschriften zu den vier Stücken verzichtet. Um sich einen Überblick über die Kompositionsstruktur und mögliche semantische Phänomene zu verschaffen, seien die beiden kürzeren mittleren Sätze genauer betrachtet. ${ }^{15}$

Die vier Eingangstakte (Molto adagio) von Nr. 2 bilden die Kernphrase und wirken wie ein Aufschwung, der durch Pausen unterbrochen sogleich wieder zum

14 JUNG, Hermann. „Minimalismus“ bei Franz Schubert. In Von Perotin bis Steve Reich. Die Ideen des ,Minimalen “ in der Musikgeschichte und Gegenwart. Bericht über das internationale Symposium Melos-Étos. Bratislava, 2005, S. 46-54.

15 Die angegebenen Taktzahlen beziehen sich auf die Ausgabe: Leoš Janáček. Klavierwerke / Piano works. BARVÍK, Miroslav - ZIMMERMANN, Reiner (eds.). Frankfurt/M., Leipzig, London, New York: [C. F. Peters], 2001. 
Abschwung wird und bis T. 16 in Varianten ausläuft. Die Presto-Takte 17-19 bilden mit dem Orgelpunkt im Bass und enggeführten, diminuierten Motivsplittern einen Kontrast dazu, anschließend (Tempo I) wird T.1-4 fast wörtlich wieder aufgenommen. Ein ebenfalls kontrastierender Presto-Teil (T. 28-38) setzt mit dem Kopf der Phrase und einer synkopierten, durch rubato und Akzentuierung gesteigerten Melodie ein, die abrupt wieder zur erneuten Variante von Tempo I zurückführt. Die kontrastierende Dur-Variante des Presto endet in T. 50 offen. Das anschließende Grave bringt eine durch espressivo und accelerando bestimmte Variante der Presto-Takte 30 ff., die wiederholt wird und danach das rasche Tempo mit figurativen Erweiterungen und Ansätzen zum doppelten Kontrapunkt (Stimmtausch) wieder aufnimmt. T. 74-85 (Tempo I) bringt erneut Varianten der T. 1, 20 und 39 und endet wiederum offen. Das abschließende Adagio (T. 86-101) wirkt wie eine Coda mit Figurationen, Floskeln und Motivteilen aus den vorangegangenen Abschnitten, sie endet in Dur auf dem Einzelton des.

Eine ähnliche Struktur, die generell in allen vier Stücken des Zyklus‘ und zudem in allen anderen Klavierwerken zwischen 1901 und 1912 zu beobachten ist, weist auch Nr. 3 auf. Was in T. 1-4 wie ein „Lied ohne Worte“ oder wie ein Klaviervorspiel zu einem realen Lied daherkommt, scheint in T. 5-7 sogleich wieder zurückgenommen. Die nachfolgenden teils figurativen, teils verarbeitenden Variationsteile speisen sich aus dem in T. 1 und T. 2 vorgestellten Achtel-Motiven als Kernmotive des „Liedes“. Die Schlussvariante (T. 69-75) bricht am Ende unvermittelt ab. Gelegentliche wörtliche Wiederholungen von Abschnitten verstärken zusätzlich den Fluss fortlaufender Veränderungen.

Die knappen, lediglich Motivik und Variantentechnik berücksichtigenden analytischen Hinweise zeigen auf der einen Seite eine klar nachzuvollziehende durchstrukturierte Faktur der Sätze. Andererseits lassen sie beim Hörer eine gewisse Ratlosigkeit als diffusen Gesamteindruck zurück. Ist es die Zerrissenheit, die durch ständige Wechsel von Dynamik und Agogik, von Tempo- und Taktangaben, von Verkürzungen und unvermittelten Abbrüchen, vom Changieren zwischen Tonarten, Tongeschlecht und farbiger Harmonik hervorgerufen wird? Sind es die vielfältigen Figurationen, Motivverarbeitungen und -varianten, die jeweils um bestimmte vorgegebene Motivkerne kreisen und den Eindruck eines Suchens nach neuen Wegen des Komponierens vermitteln? Manche Interpreten setzen solche Befunde wohl zurecht mit Janáčeks Lebenssituation und persönlicher Gestimmtheit in Beziehung, aus der Unsicherheit, tiefe Melancholie und Resignation eines Menschen spricht. ${ }^{16}$ Andere sehen darin neue Formen des Charakterstücks im 20. Jahrhundert.

16 U. a. HONOLKA, Kurt. Leoš Janáček. Sein Leben, sein Werk, seine Zeit. Stuttgart und Zürich, 1982, bes. S. 132, 147; Saremba, Leoš Janáček (Anm. 4), S. 205, 212. 
$V$ mlhách (Im Nebel): Der Titel des Klavierzyklus' wird zum Schlüssel, das Naturereignis zum Symbol einer Schaffenskrise. Mit Jenufa und der bald darauf erfolgenden Anerkennung als Opernkomponist außerhalb seiner engeren Heimat wird sich der Nebel lichten.

Hermann Jung, geb. 1943 in München; Professor für Musikwissenschaft an der Staatlichen Hochschule für Musik und Darstellende Kunst Mannheim von 1983-2008. Zahlreiche Publikationen zu Zeichen- und Symbolforschung in den Künsten, zu Musik und Sprache, zu Musik und Kultur des 17. bis 20. Jahrhunderts (insbes. zur Mannheimer Hofkapelle und zur Wiener Klassik), zur Musikgeschichte Osteuropas und zur Rezeptionsforschung (antike Mythen und Musik).

\begin{abstract}
:
The genius loci apparent at Janáček in the first decade of the $20^{\text {th }}$ century in three works for solo piano Po zarostlém chodnićku (On an Overgrown Path), two movements of Sonáta 1. X. 1905 and in $V$ mlhách (In The Mists) is based on three aspects: the biographical influences (Moravia as a home, the city of Brno, political events), the music-historical phenomena (development and transformation of compositional structures in the $19^{\text {th }}$ century) and the short analysis (technique of motif processing and variation) and semantics of the piano works. The Title $V$ mlhách becomes here the key, the natural phenomenon becomes the symbol of a creative block.
\end{abstract}

\title{
Key words:
}

biography, Brno, change in composition structures during the $19^{\text {th }}$ century, creative block, piano works, political events, semantical analysis, technique of motif processing and variation

\section{Bibliography:}

EDLER, Arnfried. Gattungen der Musik für Tasteninstrumente. (= Handbuch der musikalischen Gattungen 7,3). Laaber, 2003.

HONOLKA, Kurt. Leoš Janáček. Sein Leben, sein Werk, seine Zeit. Stuttgart und Zürich, 1982.

JANÁČEK, Leoš. Klavierwerke / Piano works. Barvík, Miroslav - Zimmermann, Reiner (eds.). Frankfurt/M., Leipzig, London, New York: [C. F. Peters], 2001.

JIRÁNEK, Jaroslav. Janáčeks Klavierkompositionen vom Standpunkt ihres dramatischen Charakters. Versuch einer semantischen Analyse. Archiv für Musikwissenschaft XXXIX, 1982, S. 179-197; zur Begrifflichkeit S. 187.

JUNG, Hermann. „Minimalismus“ bei Franz Schubert. In Von Perotin bis Steve Reich. Die Ideen des ,Minimalen " in der Musikgeschichte und Gegenwart. Bericht über das internationale Symposium Melos-Étos. Bratislava, 2005, S. 46-54.

JUNG, Hermann. Musik des ,traurigen Lächelns' - ein Virtuose wird zum Poeten. Zu den Nocturnes von Frédéric Chopin. In JUNG-KAISER, Ute - KRUSE, Matthias (Hrsg.). Chopin, der Antistar. (= Wegzeichen Musik 5). Hildesheim / Zürich etc., 2010.

KNAUS, Jakob. Intime Briefe 1879/80 aus Leipzig und Wien. Zürich, 1985.

Leoš Janáček in Briefen und Erinnerungen. Ausgewählt, mit Beiträgen und Anmerkungen versehen von Bohumír Štědroň. Prague ,1955, S. 80.

SAREMBA, Meinhard. Leoš Janáček. Zeit - Leben - Werk - Wirkung. Kassel / Basel etc., 2001.

TYRRELL, John (Hrsg.). My Life with Janáček - The Memoirs of Zdenka Janáčková. London, 1998. 